\title{
The role of Rayleigh-Taylor instabilities in filament threads
}

\author{
J. Terradas, R. Oliver, and J. L. Ballester
}

\author{
Departament de Física, Universitat de les Illes Balears, 07122 Palma de Mallorca, Spain \\ e-mail: jaume.terradas@uib.es
}

Received 13 February 2012 / Accepted 28 March 2012

\begin{abstract}
Context. Many solar filaments and prominences show short-lived horizontal threads lying parallel to the photosphere.

Aims. In this work the possible link between Rayleigh-Taylor instabilities and thread lifetimes is investigated.

Methods. This is done by calculating the eigenmodes of a thread modelled as a Cartesian slab under the presence of gravity. An analytical dispersion relation is derived using the incompressible assumption for the magnetohydrodynamic (MHD) perturbations.

Results. The system allows a mode that is always stable, independently of the value of the Alfvén speed in the thread. The character of this mode varies from being localised at the upper interface of the slab when the magnetic field is weak, to having a global nature and resembling the transverse kink mode when the magnetic field is strong. On the contrary, the slab model permits another mode that is unstable and localised at the lower interface when the magnetic field is weak. The growth rates of this mode can be very short, of the order of minutes for typical thread conditions. This Rayleigh-Taylor unstable mode becomes stable when the magnetic field is increased, and in the limit of strong magnetic field it is essentially a sausage magnetic mode.

Conclusions. The gravity force might have a strong effect on the modes of oscillation of threads, depending on the value of the Alfvén speed. In the case of threads in quiescent filaments, where the Alfvén speed is presumably low, very short lifetimes are expected according to the slab model. In active region prominences, the stabilising effect of the magnetic tension might be enough to suppress the Rayleigh-Taylor instability for a wide range of wavelengths.
\end{abstract}

Key words. magnetohydrodynamics (MHD) - waves - Sun: filaments, prominences

\section{Introduction}

Threads are thought to be the building blocks of solar filaments and prominences. These fine-scale structures are readily seen in high quality images obtained using the Swedish Solar Telescope (SST), the Dutch Open Telescope (DOT), or the Solar Optical Telescope (SOT) onboard Hinode. Threads are a part of a magnetic tube filled with cold plasma with densities much larger than the coronal surrounding, the density contrast being of the order 100 . Their observed widths are typically between $100 \mathrm{~km}$ and $600 \mathrm{~km}$, while their lengths are in the range 3500-28 $000 \mathrm{~km}$ (see Lin et al. 2005). The total length of the magnetic tube where the thread is supposed to be embedded is much longer than the thread length, and in many cases is of the order of $10^{5} \mathrm{~km}$. Some threads are essentially horizontal, i.e., running parallel to the photosphere, and are usually located along the spine of the filament (see Okamoto et al. 2007, for a clear example). Inclined threads are mostly found in filament barbs, whereas vertical threads are clearly observed in hedgerow prominences (see for example Berger et al. 2008; Chae et al. 2008).

Threads have short lifetimes, and in quiescent filaments they are in the range 9-20 min (see the review of Lin 2011). The mechanisms that produce the thread disappearance are usually thought to be related to mass flows and thermal instabilities (see for example, Carbonell et al. 2004; Soler et al. 2011, 2012, in the context of prominences). However, other types of instabilities may play a role. Recently, Ryutova et al. (2010) have shown that a number of processes taking place in prominences during their evolution can be linked to fundamental fluid instabilities such as Rayleigh-Taylor and Kelvin-Helmholtz instabilities (see also Berger et al. 2010). Using a single interface model between the prominence and corona, and the assumption of incompressibility for the perturbations, these authors have been able to attribute the appearance of plumes, spikes and ripples to these instabilities. Hillier et al. $(2011,2012)$ have numerically investigated the nonlinear stability of the Kippenhahn-Shlüter prominence model to the Rayleigh-Taylor instability.

An additional feature of threads is that they show oscillations. For example, Thompson \& Schmieder (1991); Yi \& Engvold (1991); Yi et al. (1991) found that some threads seem to oscillate independently while others appear to oscillate together (see the reviews of Oliver \& Ballester 2002; Banerjee et al. 2007; Arregui et al. 2012). More recent high spatial resolution and high cadence observations have revealed the presence of both propagating and standing waves in individual threads (see Lin et al. 2007, 2009; Okamoto et al. 2007). For example, Lin et al. (2007) found oscillations with wavelengths of the order of $3000 \mathrm{~km}$ and periods around $5.4 \mathrm{~min}$ in quiescent filaments, while Lin et al. (2009) have reported periods of $3.6 \mathrm{~min}$ in swaying filament threads. Okamoto et al. (2007) have detected waves in an active region prominence with wavelengths of at least $2 \times 10^{5} \mathrm{~km}$ with periods of $4 \mathrm{~min}$. Note that the periods of oscillation are typically of the same order of magnitude as the lifetimes of threads in quiescent filaments.

The theoretical interpretation of the reported oscillations in threads is usually done by means of magnetohydrodynamic (MHD) waves. A certain prominence model is assumed and the eigenmodes of oscillation are determined (see Oliver \& Ballester 2002; Arregui et al. 2012, for a detailed description of the results using different configurations). In particular, two basic models have been considered in the literature, the Cartesian slab (see Joarder et al. 1997; Díaz et al. 2001, 2005), and the cylindrical 
tube (see for example, Díaz et al. 2002; Dymova \& Ruderman 2005; Soler 2010; Arregui et al. 2008, 2011). MHD waves in cylindrical plasmas are characterised by two wavenumbers, the axial wavenumber and the azimuthal wavenumber. For typical threads the azimuthal wavenumber of the detected MHD waves is much larger than the longitudinal wavenumber. The advantage of the cylindrical tube is that it is more realistic than the Cartesian slab which is, in general, two dimensional and thus infinite in the third direction. However, the inclusion in the slab configuration of propagation in this third direction, equivalent to the azimuthal component in a cylindrical tube, mimics the effect of a three-dimensional structure regarding the MHD waves.

The motivation of the present work is to study oscillations of horizontal threads under the presence of the gravitational force. This problem has been partially addressed by McEwan \& Díaz (2007) using a different approach. Here the thread is modelled using the slab configuration. The incompressible assumption is adopted for the perturbations, which are allowed to be threedimensional. The eigenmodes of the system are carefully analysed, paying special attention to the regime where the system becomes unstable due to the Rayleigh-Taylor instability, driven by the gravity force. Our focus is on the possible relation between this instability and the short lifetimes of threads.

\section{Single interface results}

It is well known that a single interface between fluids with different densities, like the interface between threads and the surrounding corona, can be unstable in the presence of gravity when the upper fluid is heavier that the lower fluid, this is the Rayleigh-Taylor instability (Rayleigh 1883; Taylor 1950). Let us assume that we have an interface in the $z$-direction. A constant magnetic field, $\boldsymbol{B}_{0}$, is imposed to be along the $x$-direction, and gravity, $\boldsymbol{g}$, is acting across the magnetic field. The sketch of the configuration is represented in Fig. 1 for a double interface, but at this point we just consider a single interface. We assume that the equilibrium density, $\rho_{0}$, is constant at each side of the interface $\left(\rho_{0}=\rho_{\mathrm{p}}\right.$ for $z>0$, while $\rho_{0}=\rho_{\mathrm{c}}$ for $\left.z<0\right)$. Since the density is a piecewise constant magnitude the equilibrium gas pressure, $p_{0}$, has to change linearly with $z$ due to the presence of the gravity force, and must be continuous at the interface to have hydrostatic balance.

We use the incompressible linearised ideal MHD equations for the perturbations. We seek for solutions with a dependence on $t, x$ and $y$ of the form $\mathrm{e}^{\mathrm{i} \omega t+\mathrm{i} k_{x} x+\mathrm{i} k_{y} y}$, where $\omega$ is the frequency and $k_{x}$ and $k_{y}$ are the wavenumbers parallel and perpendicular to the magnetic field, respectively. The total wavenumber is $k=$ $\sqrt{k_{x}^{2}+k_{y}^{2}}$. The incompressibility constraint is

$\nabla \cdot \boldsymbol{v}=\mathrm{i} k_{x} v_{x}+\mathrm{i} k_{y} v_{y}+\frac{\mathrm{d} v_{z}}{\mathrm{~d} z}=0$

Under such assumption the continuity equation for the perturbed density reduces, in our configuration, to

$\mathrm{i} \omega \rho=-v_{z} \frac{\mathrm{d} \rho_{0}}{\mathrm{~d} z}$.

From the momentum equation we have

$$
\begin{aligned}
& \mathrm{i} \omega \rho_{0} v_{x}=-\mathrm{i} k_{x} p, \\
& \mathrm{i} \omega \rho_{0} v_{y}=-\mathrm{i} k_{y} p+\frac{B_{0}}{\mu}\left(\mathrm{i} k_{x} b_{y}-\mathrm{i} k_{y} b_{x}\right), \\
& \mathrm{i} \omega \rho_{0} v_{z}=-\frac{\mathrm{d} p}{\mathrm{~d} z}+\frac{B_{0}}{\mu}\left(\mathrm{i} k_{x} b_{z}-\frac{\mathrm{d} b_{x}}{\mathrm{~d} z}\right)-\rho g,
\end{aligned}
$$

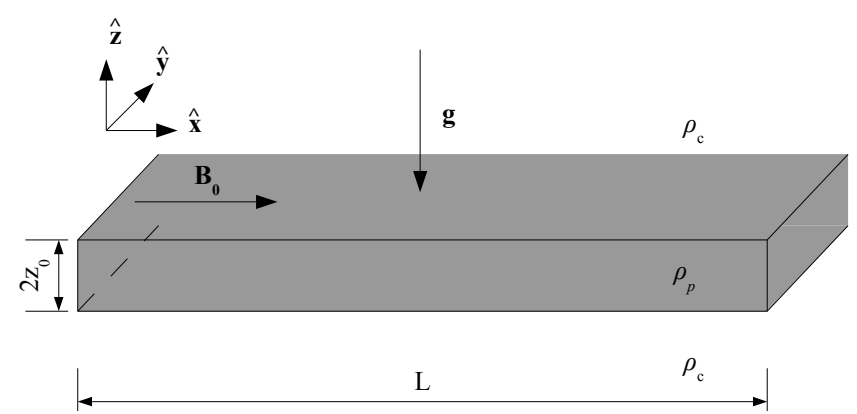

Fig. 1. Sketch of the slab model representing an idealised thread.

while from the induction equation we obtain

$\mathrm{i} \omega b_{x}=B_{0} \mathrm{i} k_{x} v_{x}$
$\mathrm{i} \omega b_{y}=B_{0} \mathrm{i} k_{x} v_{y}$
$\mathrm{i} \omega b_{y}=B_{0} \mathrm{i} k_{x} v_{z}$

Note that the equilibrium gas pressure, $p_{0}$, does not appear in the previous equations because of the incompressible assumption, but the perturbed pressure, $p$, is in the equations although we do not have an explicit equation for this magnitude.

We want to obtain a single equation for $v_{z}$, and the first step is to insert the corresponding components of the perturbed magnetic field given by Eqs. (6)-(8) in (4)-(5) and make use of Eq. (2). The following equations are obtained

$\mathrm{i} \omega \rho_{0} v_{y}=-\mathrm{i} k_{y} p+\frac{B_{0}^{2}}{\mu} \frac{k_{x}}{\omega}\left(\mathrm{i} k_{x} v_{y}-\mathrm{i} k_{y} v_{x}\right)$,
$\mathrm{i} \omega \rho_{0} v_{z}=-\frac{\mathrm{d} p}{\mathrm{~d} z}+\frac{B_{0}^{2}}{\mu} \frac{k_{x}}{\omega}\left(\mathrm{i} k_{x} v_{z}-\frac{\mathrm{d} v_{x}}{\mathrm{~d} z}\right)+\frac{1}{\mathrm{i} \omega} g \frac{\mathrm{d} \rho_{0}}{\mathrm{~d} z} v_{z}$.

Eliminating the pressure perturbation from Eqs. (3) and (9) we find that

$\mathrm{i} k_{x} v_{y}-\mathrm{i} k_{y} v_{x}=0$,

thus, the second term in the right hand side of Eq. (9) is zero. Using Eqs. (3) and (9) together with Eq. (1) we find the following expression for the perturbed gas pressure

$p=-\mathrm{i} \rho_{0} \frac{\omega}{k^{2}} \frac{\mathrm{d} v_{z}}{\mathrm{~d} z}$

In addition, combining Eqs. (1) and (11) we obtain

$v_{x}=\mathrm{i} \frac{k_{x}}{k^{2}} \frac{\mathrm{d} v_{z}}{\mathrm{~d} z}$.

Now inserting Eqs. (12) and (13) in (10) we find the final equation for $v_{z}$

$\frac{\mathrm{d}}{\mathrm{d} z}\left(\rho_{0} \frac{\mathrm{d} v_{z}}{\mathrm{~d} z}\right)+\frac{B_{0}^{2}}{\mu} \frac{k_{x}^{2}}{\omega^{2}}\left(k^{2}-\frac{\mathrm{d}^{2}}{\mathrm{~d} z^{2}}\right) v_{z}-k^{2}\left(\rho_{0}+\frac{g}{\omega^{2}} \frac{\mathrm{d} \rho_{0}}{\mathrm{~d} z}\right) v_{z}=0$.

It is easy to see that for a piecewise density profile the eigenfunctions have the following simple form

$v_{z}(z)= \begin{cases}A \mathrm{e}^{k z}, & z<0 \\ B \mathrm{e}^{-k z}, & z>0\end{cases}$

The constants $A$ and $B$ are calculated requiring continuity of $v_{z}$ across the interface (meaning that $A=B$ ). There is an additional condition that comes from the integration of Eq. (14) over an 
infinitesimal element of $z$ that includes the interface (located at $z=0)$. This condition reduces to

$$
\begin{aligned}
\left(-\omega^{2} \rho_{\mathrm{p}}+k_{x}^{2} \frac{B_{0}^{2}}{\mu}\right)\left(\frac{\mathrm{d} v_{z}}{\mathrm{~d} z}\right)^{+} & +\left(\omega^{2} \rho_{\mathrm{c}}-k_{x}^{2} \frac{B_{0}^{2}}{\mu}\right)\left(\frac{\mathrm{d} v_{z}}{\mathrm{~d} z}\right)^{-} \\
= & -g k^{2}\left(\rho_{\mathrm{p}}-\rho_{\mathrm{c}}\right) v_{z}(z=0),
\end{aligned}
$$

where we have used that $\rho_{0}=\rho_{\mathrm{p}}$ for $z>0$ ("+" region) and $\rho_{0}=\rho_{\mathrm{c}}$ for $z<0$ ("-" region). Inserting the derivatives of $v_{z}$ at each side of the interface in Eq. (16) leads to the well known dispersion relation for the incompressible interface problem (see also Chandrasekhar 1961)

$\omega^{2}=-g k \frac{\rho_{\mathrm{p}}-\rho_{\mathrm{c}}}{\rho_{\mathrm{p}}+\rho_{\mathrm{c}}}+k_{x}^{2} \frac{2 B_{0}^{2}}{\mu\left(\rho_{\mathrm{p}}+\rho_{\mathrm{c}}\right)}$.

From this equation it is clear that in the absence of magnetic field the system is always unstable $\left(\omega^{2}<0\right)$ if $\rho_{\mathrm{p}}>\rho_{\mathrm{c}}$, i.e., when the heavier fluid is on top of the lighter fluid. The opposite configuration is always stable, i.e., $\omega^{2}>0$ for $\rho_{\mathrm{p}}<\rho_{\mathrm{c}}$. The unstable mode can become stable if the second term of the right-hand side of Eq. (17) is larger than the absolute value of the first term. This term is simply the square of the kink frequency. It is interesting to note that Eq. (17) simplifies to

$\omega^{2}=-g k+k_{x}^{2} \frac{2 B_{0}^{2}}{\mu \rho_{\mathrm{p}}}$,

if the density at one side of the interface is much larger that the density at the other side ( $\rho_{\mathrm{p}} \gg \rho_{\mathrm{c}}$, in the previous equation). This is a common characteristic in prominence threads.

\section{Slab model results}

The interface model is useful to understand the basics of the Rayleigh-Taylor instability. However, the main purpose of this work is to study threads, and these structures are better represented using slab or cylindrical models. Here we assume a slab configuration. Across the field, i.e., in the $z$-direction, we assume again that density is constant. The equilibrium gas pressure is chosen to have hydrostatic equilibrium, and as in the interface model it changes linearly with $z$. The density takes the value $\rho_{\mathrm{p}}$ inside the slab, representing a thread located between $-z_{0}$ and $z_{0}$, and $\rho_{\mathrm{c}}$ outside the slab (see Fig. 1). In this configuration the Alfvén speed has a constant value inside and outside the thread. Notice that the thread is infinite in the $y$-direction. The model adopted here is similar to that studied in McEwan \& Díaz (2007), where the main distinctive difference is the incompressible assumption for the MHD waves and specially the treatment of the boundary conditions at the interfaces, given in our case by Eq. (16). Goedbloed \& Poedts (2004) analysed a gravitating slab supported from below by a vacuum magnetic field but conducting wall boundary conditions were considered (see also the works of Hermans \& Goossens 1987, 1989).

The slab configuration is essentially the combination of two interfaces. Extending the single interface eigenfunction, given by Eq. (15), to the double interface problem, leads to

$v_{z}(z)= \begin{cases}A \mathrm{e}^{k\left(z+z_{0}\right)}, & z<-z_{0}, \\ B \mathrm{e}^{-k\left(z+z_{0}\right)}+C \mathrm{e}^{k\left(z-z_{0}\right)}, & z \leq\left|z_{0}\right|, \\ D \mathrm{e}^{-k\left(z-z_{0}\right)}, & z>z_{0} .\end{cases}$

Continuity of $v_{z}$ at $z= \pm z_{0}$ imposes a relation between constants

$B=A-C \mathrm{e}^{-2 k z_{0}}$,

$D=C+B \mathrm{e}^{-2 k z_{0}}$
Since one of these constants can be chosen arbitrarily we assume that $A=1$. The constant $D$ is written, using the equation for $B$, as a function of the constant $C$ only. The next step is to apply the second boundary condition, given by Eq. (16), at each interface $\left(z= \pm z_{0}\right)$ using the appropriate values for the densities. These boundary conditions provide two equations for $\omega^{2}$ in terms of the constant $C$, which is still unknown. The explicit form of this is calculated by combining these two equations.

There are two solutions for $C$ representing two different types of modes, hereafter denoted by the subscripts "+" and "-". The final dispersion relation is the following algebraic equation

$\omega^{2}=\frac{-g k\left(\rho_{\mathrm{p}}-\rho_{\mathrm{c}}\right)+k_{x}^{2} \frac{2 B_{0}^{2}}{\mu}\left(1-C_{ \pm} \mathrm{e}^{-2 k z_{0}}\right)}{\rho_{\mathrm{p}}\left(1-2 C_{ \pm} \mathrm{e}^{-2 k z_{0}}\right)+\rho_{\mathrm{c}}}$,

where

$C_{ \pm}=\frac{E \pm F}{G}$

being

$E=\mathrm{e}^{2 k z_{0}} g k\left(1+\frac{\rho_{\mathrm{c}}}{\rho_{\mathrm{p}}}\right) \sinh 2 k z_{0}-k_{x}^{2} v_{\mathrm{Ap}}^{2}$,

$$
\begin{aligned}
F= & \mathrm{e}^{2 k z_{0}}\left\{\left(k_{x}^{2} v_{\mathrm{Ap}}^{2}\right)^{2}\right. \\
& \left.+g^{2} k^{2}\left[\left(1+\frac{\rho_{\mathrm{c}}^{2}}{\rho_{\mathrm{p}}^{2}}\right) \sinh ^{2} 2 k z_{0}+\frac{\rho_{\mathrm{c}}}{\rho_{\mathrm{p}}} \sinh 4 k z_{0}\right]\right\}^{1 / 2},
\end{aligned}
$$

and

$G=2 \sinh 2 k z_{0}\left(k_{x}^{2} v_{\mathrm{Ap}}^{2}+g k\right)$.

In the previous expressions we have introduced the Alfvén speed in the thread, $v_{\mathrm{Ap}}=B_{0} / \sqrt{\mu \rho_{\mathrm{p}}}$. It is clear that the slab dispersion relation given by Eq. (22) has some similarities with the interface dispersion relation (Eq. (17)), but it is more involved due to the dependence of $C$ on the different parameters. For a better understanding of the solutions some limiting cases are investigated in the following subsections.

Before we analyse the different cases we need to discuss the values of some of the parameters that appear in the dispersion relation. Although we are considering an infinite slab model in the $y$-direction we incorporate the three-dimensionality of the motion by choosing the appropriate $k_{y}$. This is an important parameter in our model. If we suppose that the thread is a magnetic tube of circular cross-section then $k_{y}=m / R$ where $m$ is the azimuthal wavenumber and $R$ is the radius of the thread. For transverse motions $m=1$ (since the system is periodic in the azimuthal direction $m$ is restricted to be an integer). The equivalent mode in the slab has $k_{y}=1 / z_{0}\left(z_{0}\right.$ in the slab is equivalent to $R$ in the cylinder) and corresponds to a transverse motion of the tube if $k_{x} \ll k_{y}$. The longitudinal wavenumber is $k_{x}=2 \pi / \lambda$, being $\lambda$ the characteristic wavelength. It is clear that $k_{x} / k_{y}$ is of order $R / \lambda$. The typical radius of threads is $R \sim 10^{2} \mathrm{~km}$, while the reported wavelengths are in the range $\lambda=10^{3}-10^{5} \mathrm{~km}$. This means that the regime of interest is when $R / \lambda \ll 1$, i.e. in the thin tube approximation.

\subsection{Purely magnetic case}

We consider first the case when gravity is absent and we analyse the properties of the eigenmodes. The system has symmetric or antisymmetric eigenfunctions, meaning that either $B=C$ 

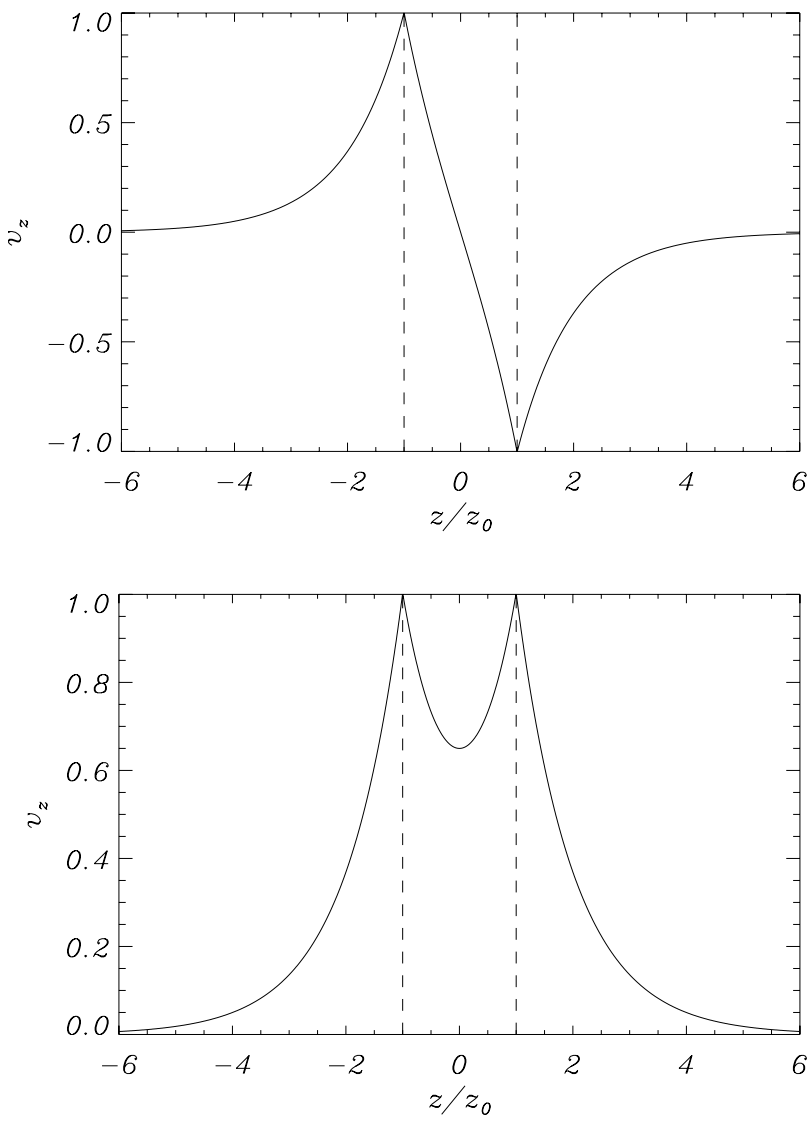

Fig. 2. Sausage (top panel) and kink (bottom panel) eigenfunctions in the purely magnetic case. In this plot $R=10^{2} \mathrm{~km}, \lambda=2 \times 10^{5} \mathrm{~km}$, $\rho_{\mathrm{p}}=100 \rho_{\mathrm{c}}, v_{\mathrm{Ap}}=100 \mathrm{~km} \mathrm{~s}^{-1}$ and $g=0$. The dashed lines represent the slab boundaries.

(for the "+" solution) or $B=-C$ (for the "-" solution), and the eigenfunctions inside the slab are the hyperbolic functions "cosh" or "sinh", respectively. The symmetric mode, or kink, satisfies that $D=A$, while for the antisymmetric or sausage mode $D=-A$. In Fig. 2 the eigenfunctions are plotted for a particular choice of parameters representative of thread conditions, $R=10^{2} \mathrm{~km}\left(k_{y}=1 / R\right), \lambda=2 \times 10^{5} \mathrm{~km}\left(k_{x}=2 \pi / \lambda\right)$, $\rho_{\mathrm{p}}=100 \rho_{\mathrm{c}}$, and $v_{\mathrm{Ap}}=100 \mathrm{~km} \mathrm{~s}^{-1}$. Contrary to the eigenmodes in a cylindrical tube (see Spruit 1982; Edwin \& Roberts 1983; Cally 1986, 2003; Goossens et al. 2009), the sausage mode in the slab model is trapped, instead of leaky. Additionally, the eigenfunction of the kink mode has a minimum at the centre of the slab instead of a maximum, but since the mode essentially produces a displacement of the whole slab, we sill refer to this mode as the transverse mode (see also Arregui et al. 2007, for the spatial distribution of the eigenfunctions in the compressional problem).

It can be shown from Eqs. (23)-(26) that in the case of zero gravity the constants are $C_{ \pm}=1 /\left(\mathrm{e}^{-2 k z_{0}} \pm 1\right)$, and the modes are always stable. The dispersion relation without gravity reduces to

$\omega^{2}=k_{x}^{2} \frac{B_{0}^{2}}{\mu} \frac{1+\operatorname{coth}\left(k z_{0}\right)}{\rho_{\mathrm{p}}+\rho_{\mathrm{c}} \operatorname{coth}\left(k z_{0}\right)}$,

for the kink mode and

$\omega^{2}=k_{x}^{2} \frac{B_{0}^{2}}{\mu} \frac{1+\tanh \left(k z_{0}\right)}{\rho_{\mathrm{p}}+\rho_{\mathrm{c}} \tanh \left(k z_{0}\right)}$, for the sausage mode. These equations agree with the results of Edwin \& Roberts (1982) when $k_{y}=0$ and in the incompressible limit (see their Eq. (12)). When $k z_{0}$ is large the hyperbolic tangent and cotangent tend to one, and we recover the dispersion relation for a single interface (see Eq. (17)) when gravity is absent, i.e., the frequency tends to the kink frequency. For a finite slab and when $k_{x} \ll k_{y}$ the frequency of these modes tends again to the kink frequency (this is true even for the compressible case, see Arregui et al. 2007), and this is the regime we are interested in.

\subsection{Purely gravitational case}

We now analyse the situation when gravity is present and $B_{0}=$ 0 . For this case it can be shown that Eq. (22) simplifies to the following dispersion relation

$$
\omega^{2}= \pm \frac{g k\left(\rho_{\mathrm{p}}-\rho_{\mathrm{c}}\right) \sinh 2 k z_{0}}{\sqrt{\left(\rho_{\mathrm{p}}^{2}+\rho_{\mathrm{c}}^{2}\right) \sinh ^{2} 2 k z_{0}+\rho_{\mathrm{p}} \rho_{\mathrm{c}} \sinh 4 k z_{0}}} .
$$

This configuration has a stable mode always associated to $C_{+}$, and an unstable solution linked to the $C_{-}$constant. The single non-magnetic interface dispersion relation, given by Eq. (18) without the magnetic term, is easily recovered from Eq. (29) when $\rho_{\mathrm{p}} \gg \rho_{\mathrm{c}}$ (the second term in the denominator can be neglected in front of the first term). If $k z_{0} \gg 1$ the dispersion relation tends also to the interface solution since the wavelength of the perturbation is much smaller than the half width of the slab. The spatial distribution of the corresponding eigenfunctions is rather different respect to the kink and sausage magnetic eigenfunctions. It is possible to see that the stable mode is mainly localised at the upper interface since for this mode $C \simeq D\left(C_{+}\right.$tends to $\mathrm{e}^{-2 k z_{0}}$ according to Eqs. (23)-(26)), while the unstable mode satisfies that $B \simeq A\left(C_{-}\right.$tends to 0$)$, and it is therefore located at the lower interface (in this case the heavier fluid is on top of the lighter fluid and for this reason the mode is unstable). An example of the eigenfunctions is found in Fig. 3. The degree of localisation of the eigenfunction around the interfaces is given by the penetration length, which according to Eq. (19), can be defined as $l=1 / k$. For the situation $k_{x} \ll k_{y}$ and $k_{y}=1 / z_{0}$ we have that $l \approx z_{0}\left(k \approx k_{y}\right)$. Hence, for these parameters, slab eigenmodes due to gravity do not represent a coherent motion of the whole slab like in the kink mode, and are mostly surface waves associated to the individual interfaces.

\subsection{Full case}

When gravitational and magnetic forces are present at the same time we expect to find modes with mixed character. As in the interface problem, the interplay of these two restoring forces might cause the unstable mode to become stable if the magnetic field is strong enough, or if the wavelength is larger than a critical value. This behaviour is associated to the $C_{-}$solution in our notation. On the contrary, the stable mode found in the situation with gravity only, remains always stable when the magnetic field is different from zero. In this case the solution corresponds to the $C_{+}$constant. An example of this behaviour is found in Fig. 4, where the square of the frequency calculated using the full dispersion relation given by Eq. (22), is plotted as a function of the wavenumber. We identify the two modes, the stable solution (dashed line) and the initially unstable mode (continuous line) that becomes stable when the wavenumber is larger than a critical value. We have used the same choice of parameters, meaning 
J. Terradas et al.: Rayleigh-Taylor instabilities in threads
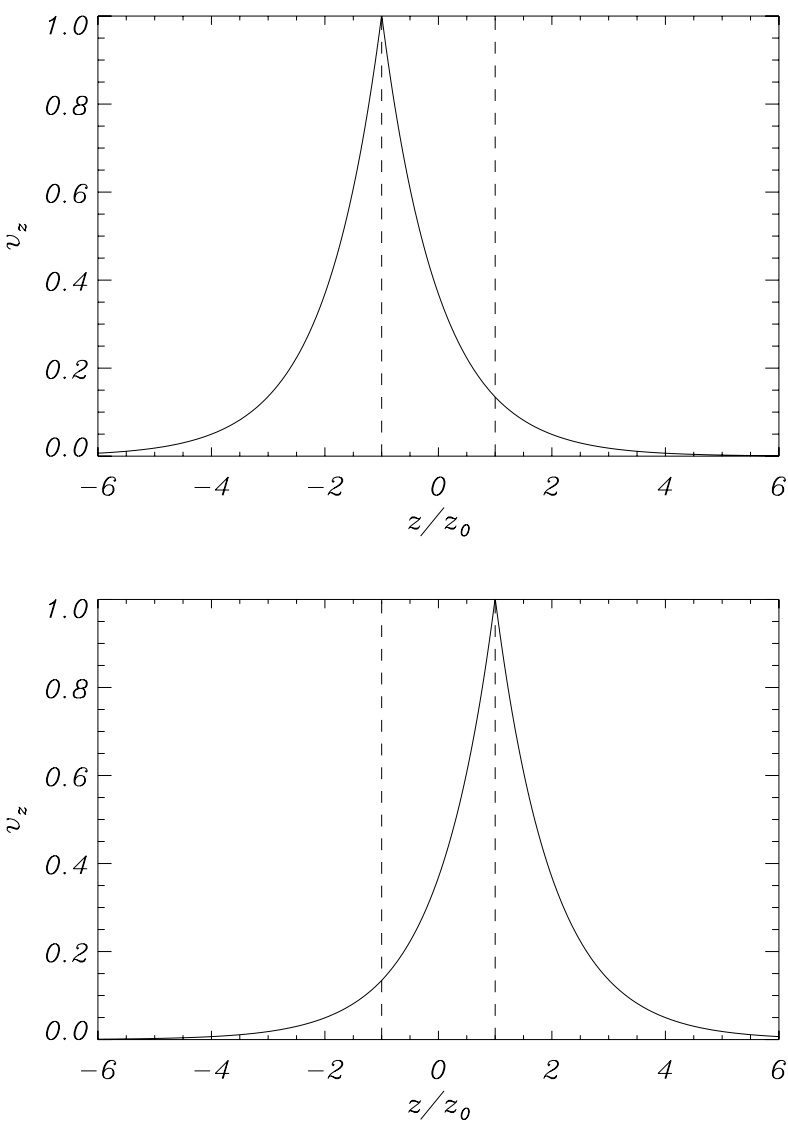

Fig. 3. Eigenfunction of the modes associated to the lower unstable mode (top panel) and upper stable mode (bottom panel) in the purely gravitational case. In this plot $R=10^{2} \mathrm{~km}, \lambda=2 \times 10^{5} \mathrm{~km}, \rho_{\mathrm{p}}=100 \rho_{\mathrm{c}}$ and $v_{\mathrm{Ap}}=0\left(g=0.274 \mathrm{~km} \mathrm{~s}^{-2}\right)$. The dashed lines represent the slab boundaries.

that we are in the regime $k_{x} \ll k_{y}$. The solutions associated to the individual interfaces, calculated using Eq. (17), are also plotted in Fig. 4 with thin lines. The agreement between the slab and interface solutions is quite good for small $k_{x}$, meaning that for the particular parameters we are using, the modes are, to all practical purposes, surface waves associated to the individual interfaces in this regime. Thus, for $k_{x}$ small there are no global kink-like transverse modes. Further confirmation of this behaviour is found in the eigenfunctions, represented in Fig. 5 for $v_{\text {Ap }}=100 \mathrm{~km} \mathrm{~s}^{-1}$ and $\lambda=2 \times 10^{4} \mathrm{~km}$ (see continuous lines). The surface waves are barely affected by the presence of the other interface, and the overall structure is quite similar to the one found in the nonmagnetic case (compare with Fig. 3).

The weakly coupled behaviour of the two interfaces manifested in Fig. 5 is better understood by analysing the value of $C$ in the dispersion relation. If we assume that $\rho_{\mathrm{c}} \ll \rho_{\mathrm{p}}$ the terms proportional to $\rho_{\mathrm{c}} / \rho_{\mathrm{p}}$ can be neglected in Eqs. (24) and (25). It is easy to see that with this simplification, and when the following condition is satisfied

$g k \sinh 2 k z_{0} \gg k_{x}^{2} v_{\mathrm{Ap}}^{2}$,

the constants $E$ and $F$ tend to same value. This means that for the unstable mode the constant $C_{-}$goes to zero, and thus for this mode the slab dispersion relation tends to the interface dispersion relation given by Eq. (18). The equivalent result is found for the stable mode. Therefore, if the condition given by Eq. (30) is satisfied, the system is well described by the interface results. Note that Eq. (30) does not mean that the role of the magnetic

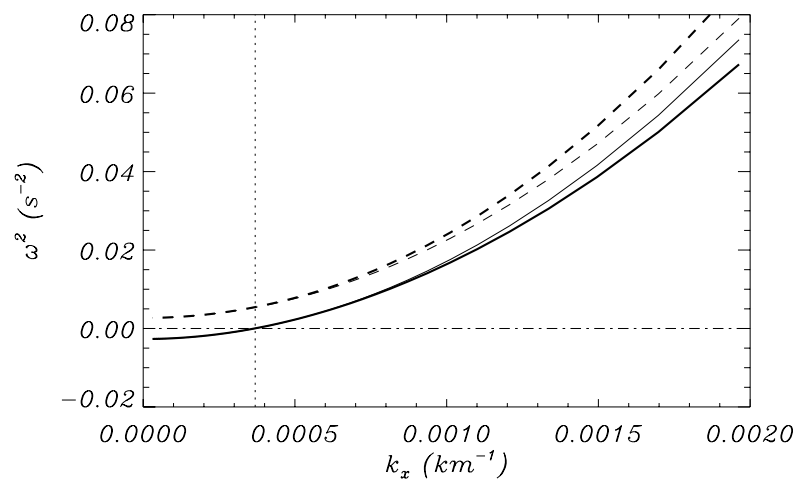

Fig. 4. Square of the frequency as a function of $k_{x}$. In this plot $R=$ $10^{2} \mathrm{~km}, \rho_{\mathrm{p}}=100 \rho_{\mathrm{c}}$, and $v_{\mathrm{Ap}}=100 \mathrm{~km} \mathrm{~s}^{-1}$. Thick lines correspond to the full dispersion relation for the slab (Eq. (22)), while thin lines represent the solutions of the single interface (Eq. (17)). The dashed lines correspond to the $C_{+}$solution while the solid lines represent the $C_{-}$solution.The dotted vertical line represents the critical wavenumber calculated using Eq. (32).

field is neglected in front of the gravitational term, the interface dispersion relation still contains the magnetic term. In this regime we can make further analytical progress assuming that $k_{x} \ll k_{y}$, and reducing Eq. (18), for the unstable mode, to

$\omega^{2}=-\frac{g}{R}+2 k_{x}^{2} v_{\mathrm{Ap}}^{2}$,

where we have used that $k_{y}=1 / z_{0}=1 / R$.

If we assume that the thread radius and the Alfvén speed are known from observations, we can determine the critical wavenumber required to have a stable configuration $\left(\omega^{2}>0\right)$

$k_{x}^{c}=\sqrt{\frac{1}{2} \frac{g}{R}} \frac{1}{v_{\mathrm{Ap}}}$.

In Fig. 4 this critical wavenumber is represented by a vertical dotted line. Alternatively, if the wavenumber is known the Alfvén speed required to have a stable configuration is

$v_{\mathrm{Ap}}^{c}=\sqrt{\frac{1}{2} \frac{g}{R}} \frac{1}{k_{x}}$.

This equation might be useful for some applications, as we will show in Sect. 4.

The condition given by Eq. (30) is not fully satisfied if, for example, we chose a wavelength of the order of $\lambda \sim 6 \times 10^{3} \mathrm{~km}$, and we consider that the Alfvén speed is $v_{\text {Ap }}=100 \mathrm{~km} \mathrm{~s}^{-1}$, i.e. when we move to the right in the dispersion diagram shown in Fig. 4. For these parameters the modes are no longer pure surface waves associated to the interface and have a mixed nature, as can be appreciated in Fig. 5 (see dotted lines). In fact in this figure the particular choice of parameters satisfies that

$g k \sinh 2 k z_{0} \approx k_{x}^{2} v_{\mathrm{Ap}}^{2}$.

This condition means that the coupling between gravitational and magnetic forces is strong, and the resulting modes a mixture between interface and slab waves. The situation is intermediate between the purely gravitational and the purely magnetic modes. Finally, when

$g k \sinh 2 k z_{0} \ll k_{x}^{2} v_{\mathrm{Ap}}^{2}$,

the modes have a global nature and are very similar to the sausage and kink modes (see Fig. 5, dash-dotted lines) since they 

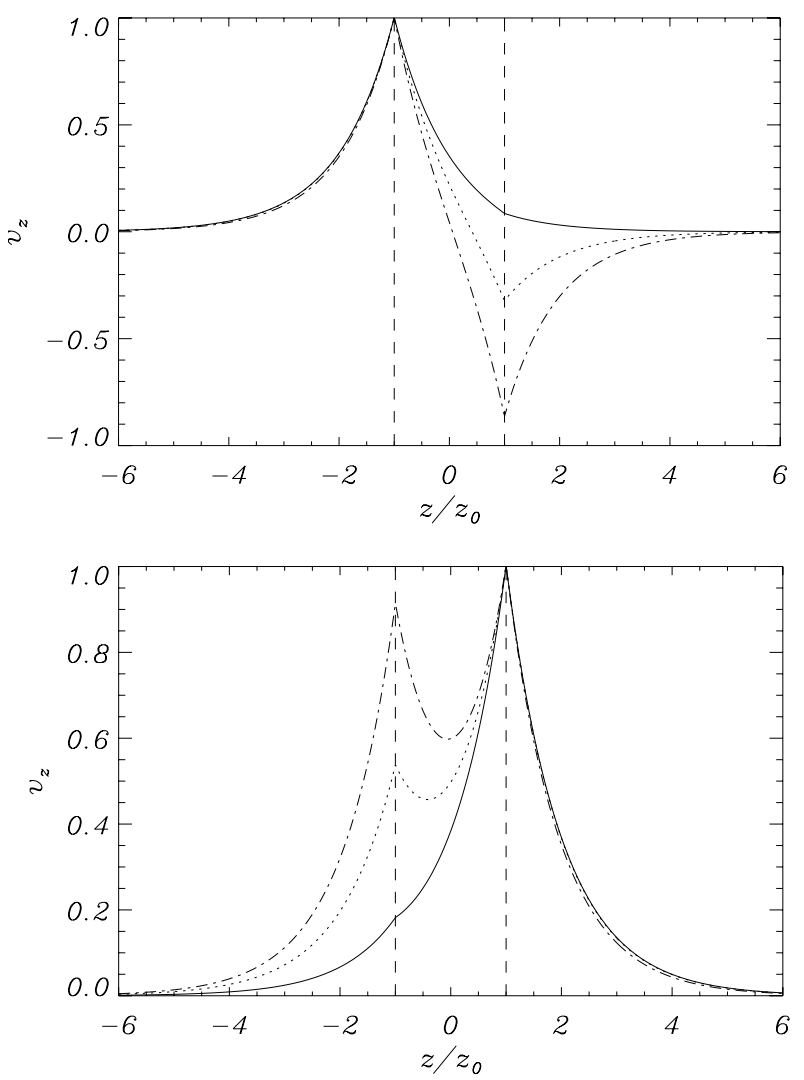

Fig. 5. Eigenfunctions in the presence of gravity and magnetic field associated to the $C_{-}$solution (top panel) and the $C_{+}$solution (bottom panel). The continuous line correspond s to $\lambda=2 \times 10^{4} \mathrm{~km}$, the dotted line to $\lambda=6 \times 10^{3} \mathrm{~km}$, while for the dash-dotted curve $\lambda=2 \times 10^{3} \mathrm{~km}$. In this plot $R=10^{2} \mathrm{~km}, \rho_{\mathrm{p}}=100 \rho_{\mathrm{c}}$ and $v_{\mathrm{Ap}}=100 \mathrm{~km} \mathrm{~s}^{-1}$. The dashed lines represent the slab boundaries.

are dominated by magnetic forces. This case is almost equivalent to the purely magnetic situation discussed in Sect. 3.1, and the modes are always stable.

Figure 5 shows very clearly the transformation from single surface waves to global sausage and kink modes when the Alfvén speed is fixed and the wavelength is allowed to change, but a completely equivalent behaviour is found if the wavelength is fixed and the Alfvén speed varies. Note also that according to the dispersion diagram given by Fig. 4 we find that the solution associated to the $C_{-}$constant changes from unstable to stable, and the eigenfunction evolves from an interface solution associated to the lower interface in the gravitationally dominated case to a sausage mode in the magnetically dominated situation. On the other hand, the solution associated to the upper interface, the $C_{+}$mode, is always stable and it eventually tends the global kink mode.

\subsection{Thread lifetimes}

From the previous results we conclude that in case of instability it will be triggered at the bottom of the thread and it is due to the $C_{-}$solution. The development of this instability will eventually produce significant changes in the whole thread structure. Here we want to calculate the time required to observe the effects on the upper interface. We know that the velocity of the lower interface is (in the linear regime)

$v=v_{0} \mathrm{e}^{t / \tau}$,

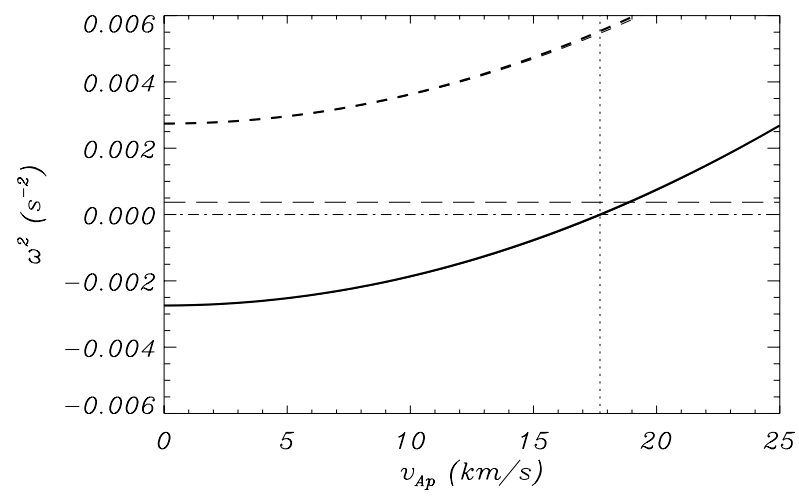

Fig. 6. Square of the frequency as a function of the Alfvén speed in the thread. In this plot $R=10^{2} \mathrm{~km}, \lambda=3 \times 10^{3} \mathrm{~km}$ and $v_{\mathrm{Ap}}^{c} \approx 17.7 \mathrm{~km} \mathrm{~s}^{-1}$. The same notation as in the previous figures has been used. The long dashed line corresponds to the frequency $(P=5.4 \mathrm{~min})$ derived from the observations of Lin et al. (2007). The dashed lines correspond to the $C_{+}$solution while the solid lines represent the $C_{-}$solution.

where $\tau=1 /|\omega|$ is the growth rate of the instability. If the interface moves upward then the vertical displacement is

$\xi=v_{0} \tau \mathrm{e}^{t / \tau}+\xi_{0}$.

We assume that at $t=0$ the interface is located at $z=-z_{0}$ (according to our slab model), this means that

$\xi_{0}=-\left(z_{0}+v_{0} \tau\right)$.

Now using Eq. (37) we can calculate the time required to have a displacement of the interface from $-z_{0}$ to $z_{0}$

$t_{\mathrm{m}}=\tau \ln \left(\frac{2 z_{0}}{v_{0} \tau}+1\right)$.

This parameter provides a rough measure of the time to blend the lower and upper interfaces of the thread and, as a result of this process there is a mixture of the thread density with the much lower coronal density. The time scale $t_{\mathrm{m}}$ can be interpreted as the disappearance time or lifetime of the thread due to the RayleighTaylor instability. However, we have to bear in mind that the previous calculation is based on the assumption of a linear regime, but in a real situation the nonlinear effects might increase the lifetimes due to the saturation of the instability.

\section{Application to observations of oscillating threads}

Lin et al. (2007) found travelling waves in a quiescent filament from the analysis of velocity Dopplergrams. The observed threads have a radius of the order of $100 \mathrm{~km}$, and their typical lifetime is $9 \mathrm{~min}$. For the particular thread marked with the label L2 in Lin et al. (2007), the period is $5.4 \mathrm{~min}$, the estimated wavelength of the propagating waves is $3000 \mathrm{~km}$, and the phase speed is around $9.5 \mathrm{~km} \mathrm{~s}^{-1}$. The amplitude of the oscillations is $1-2 \mathrm{~km} \mathrm{~s}^{-1}$.

We concentrate first on the travelling wave. Since the wave has a propagating character we interpret this mode, according to our model, as a stable solution, the unstable mode simply grows with time and it does not propagate along the thread producing oscillations (in the absence of longitudinal flows). The thread radius and the wavelength of the perturbation are known from observations, therefore we calculate the critical Alfvén speed to have stable solutions. From Eq. (33) it is found that $v_{\text {Ap }}^{c} \approx 17.7 \mathrm{~km} \mathrm{~s}^{-1}$. This is the lower bound to have stable 
J. Terradas et al.: Rayleigh-Taylor instabilities in threads

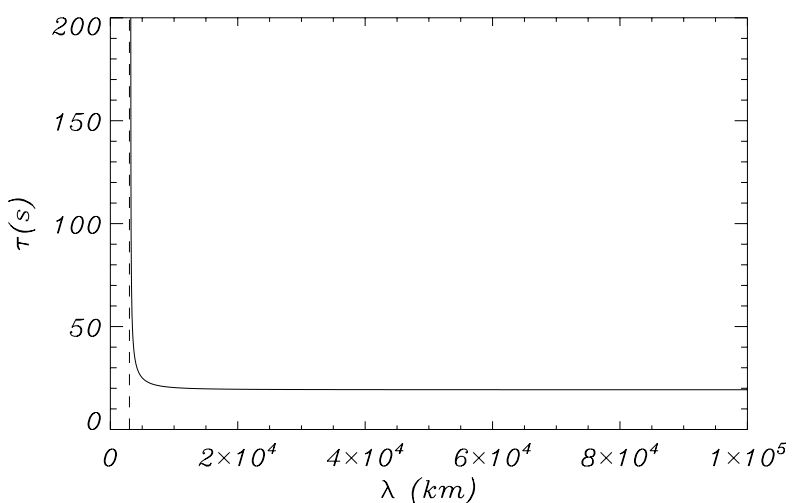

Fig. 7. Growth rate $(\tau=1 /|\omega|)$ as a function of the wavelength of the perturbation. In this plot $R=10^{2} \mathrm{~km}$ and $v_{\mathrm{Ap}}=18.8 \mathrm{~km} \mathrm{~s}^{-1}$. The dashed line denotes the wavelength, $\lambda_{\mathrm{c}}=3.2 \times 10^{3} \mathrm{~km}$, where the mode changes from stable to unstable.

modes whose wavelength is equal to or smaller than $3000 \mathrm{~km}$. The Alfvén speed in the thread might be higher than $17.7 \mathrm{~km} \mathrm{~s}^{-1}$, and the exact value is calculated using the period of oscillation known from observations, and the dispersion relation. From the two possible modes, only the solution associated to the lower interface can be matched with the parameters determined from observations. Figure 6 shows the dependence of the square of the frequency with the Alfvén speed in the thread. The Alfvén speed that produces a match with the period of $5.4 \mathrm{~min}$ (and the corresponding phase speed of $9.5 \mathrm{~km} \mathrm{~s}^{-1}$ ) is $18.8 \mathrm{~km} \mathrm{~s}^{-1}$, and has been calculated using Eq. (31). This value corresponds to the intersection of the long dashed line, representing the reported period, and the thick continuous line associated to the dispersion relation. Note that the difference between the critical and the estimated Alfvén speed in the thread is rather small.

Once we have an estimation of the Alfvén speed in the thread we calculate the critical wavelength to have a stable situation. Using Eq. (32) we obtain a value of $3200 \mathrm{~km}$. This value is slightly larger than the wavelength of the propagating wave, $3000 \mathrm{~km}$. In Fig. 7 the growth rate is plotted as a function of the wavelength, being the lower value the critical wavelength. Close to the critical wavelength the growth times can be very large but for $\lambda \geq 10^{4} \mathrm{~km}$ the growth rate is almost constant with a value of $20 \mathrm{~s}$. Using this growth rate, the value of $z_{0}$, and the value of the amplitude $v_{0}$ assumed to be of the order of $1 \mathrm{~km} \mathrm{~s}^{-1}$, we estimate from Eq. (39) that the mixing time is $t_{\mathrm{m}}=48 \mathrm{~s}$. This value is one order of magnitude smaller than the estimated lifetime of the thread, which is around $540 \mathrm{~s}$ (9 min). In this particular case the instability is too efficient and according to our model this thread should disappear in less than one minute.

Another example of thread oscillations has been investigated by Okamoto et al. (2007). Using Hinode observations these authors found transverse motions of threads in an active region prominence. Terradas et al. (2008) performed a seismological analysis of the same event. The radius of the threads are in the range $180-330 \mathrm{~km}$ and their length is $1700-16000 \mathrm{~km}$. The estimated minimum length of the magnetic tube is $L=10^{5} \mathrm{~km}$. We concentrate on the thread of length $16000 \mathrm{~km}$ since this structure has the smallest longitudinal motion (the horizontal flow velocity is around $15 \mathrm{~km} \mathrm{~s}^{-1}$ ). Contrary to the situation studied by Lin et al. (2007) the oscillations seem to have a standing nature, and the thread is moving vertically with a period of $4 \mathrm{~min}$. Another difference with respect to Lin et al. (2007) is that we have information about the maximum wavelength that satisfies the line-tying conditions. It corresponds to the fundamental mode

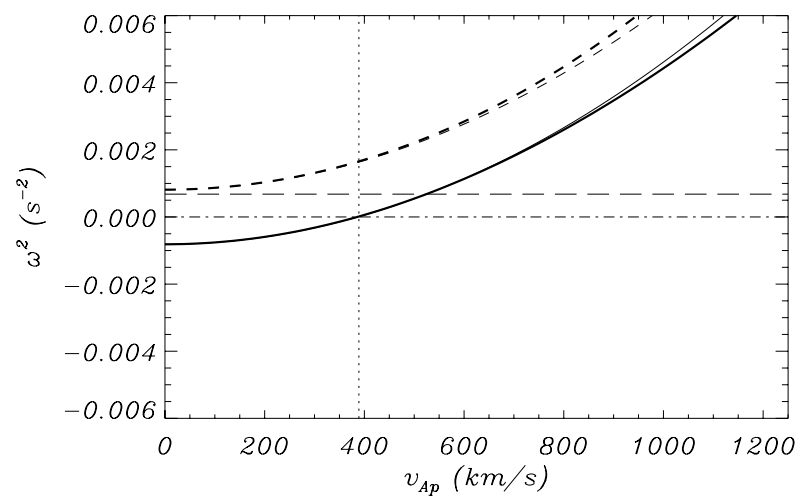

Fig. 8. Square of the frequency as a function of the Alfvén speed in the thread. In this plot $R=3.3 \times 10^{2} \mathrm{~km}, \lambda=1.2 \times 10^{5} \mathrm{~km}$ and $v_{\mathrm{Ap}}^{c} \approx$ $389.1 \mathrm{~km} \mathrm{~s}^{-1}$. The same notation as in the previous figures has been used. The long dashed line corresponds to the frequency $(P=4 \mathrm{~min})$ derived from the observations of Okamoto et al. (2007). The dashed lines correspond to the $C_{+}$solution while the solid lines represent the $C_{-}$solution.

$(\lambda=2 L)$ meaning that for a fully filled thread $\lambda=2 \times 10^{5} \mathrm{~km}$. However, we can take into account the fact that the tube is only partially filled. Soler et al. (2010) calculated the frequency of oscillation for a thread of length $L_{\mathrm{p}}$ (the total length of the tube is $L$ ). From the comparison of this frequency with the frequency of oscillation of the fully filled thread they found that the effective wavelength that should be used is $\lambda=\pi \sqrt{\left(L-L_{\mathrm{p}}\right) L_{\mathrm{p}}}$. This expression is valid as long as $L_{\mathrm{p}} / L<0.4$. For the thread we are considering we have $L_{\mathrm{p}}=16000 \mathrm{~km}$ while $L=10^{5} \mathrm{~km}$, $\left(L_{\mathrm{p}} / L=0.16\right)$, and the corresponding effective wavelength is $\lambda=1.2 \times 10^{5} \mathrm{~km}$. This is a simple way to mimic the effect of a partially filled tube. Using this effective value for the wavelength we calculate from Eq. (33) that the critical Alfvén speed to have a stable situation is $389.1 \mathrm{~km} \mathrm{~s}^{-1}$. Therefore, if the Alfvén speed is larger than this value the thread is going to be always stable with respect to the Rayleigh-Taylor instability. Figure 8 shows the dependence of the square of the frequency with the Alfvén speed in the thread for the previous example. Again, as for the situation studied in Lin et al. (2007) only the mode associated to the lower interface can be fitted with the reported period, and the inferred Alfvén speed in the thread is $525.8 \mathrm{~km} \mathrm{~s}^{-1}$. This speed is in the range of values estimated by Terradas et al. (2008). However, the interpretation in terms of the interface mode does not agree with observations since for this particular example the whole thread shows a clear kink-like vertical motion. Again the effect of gravity seems to be too strong in the slab model.

\section{Discussion and conclusions}

In this work threads have been modelled as Cartesian slabs. The presence of gravity acting perpendicularly to the axis of the structure does not allow us to have a simple equilibrium using a more appropriate geometry. The most elemental models in cylindrical geometry involve the presence of magnetic twist (see for example Lerche \& Low 1980; Low \& Zhang 2004; Petrie et al. 2007; Blokland \& Keppens 2011a). To simplify things and to have an analytical treatment of the problem, instead of a thread with cylindrical cross-section we have adopted the slab configuration. We have supposed that the slab is infinite in the ignorable direction, and more important, we have assumed that there is 


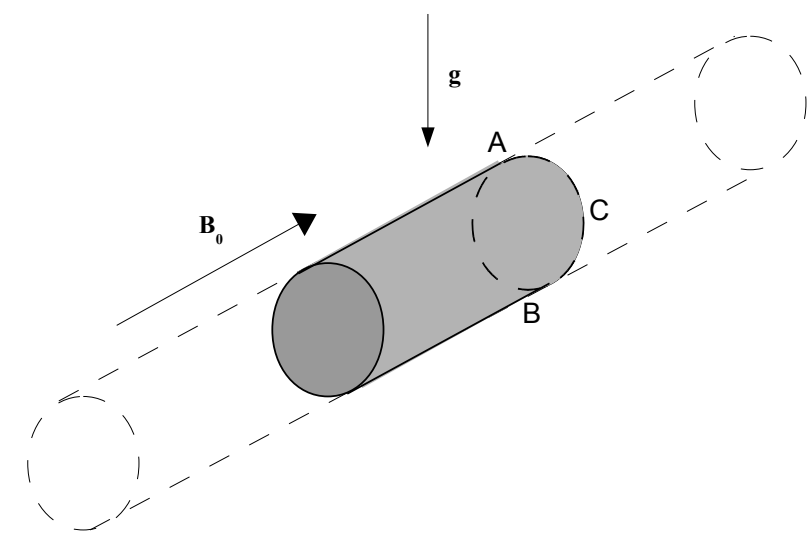

Fig. 9. Sketch of the three-dimensional cylindrical model representing a prominence thread. The projection of the gravity force varies along the circular interface.

dense material along the whole tube since the gravity force prevents to have a simple analytical equilibrium between the dense and the evacuated part of the thread along the axial magnetic field. These features of our model certainly affect the frequencies of oscillation of the stable modes and the growth rates of the unstable modes. In addition, the effect of gravity is overestimated in the slab configuration because the gravity force is perpendicular to all the points on the interfaces separating the two fluids (see Fig. 1). In a more realistic three-dimensional model, such as a cylindrical tube, the overall contribution of the gravity force perpendicular to the interface is weaker. This can be understood from Fig. 9, gravity is perpendicular to the interface only at points $A$ and $B$, while at point $C$ the projection across the interface is zero. However, we have to bear in mind that a realistic geometry could be anything between a slab and a flattened cylinder, which might be closer to a slab of finite width rather than to a pure circular cross-section cylinder.

We have shown that the mode that fits with the frequency reported in the observations is always associated, according to our model, to the lower interface and does not have a global nature. This is in contradiction with the observed motions of Okamoto et al. (2007) displaying a global vertical displacement of the whole thread. If the effect of gravity on the eigenfrequencies is smaller, as we expect for a more realistic configuration, then a match in frequency for the global mode is possible (the upper curve in Fig. 8 might intersect the dashed line). Additionally, the growth rate of the unstable mode will increase, which is one of the questionable results of the slab model since the growth of the instability is too fast, according to the comparison with the observations of Lin et al. (2007).

We have also adopted in our analysis the incompressible assumption for the perturbations. It is known that, in general, the effect of compressibility helps to stabilise the unstable modes. Since gas pressure must change with position to have hydrostatic equilibrium, the analytical investigation of the compressible case is much more complicated than the incompressible situation due to the presence on non-constant coefficients in the equations (for example, the sound speed would change across the slab). However, even under the compressible assumption, in the regime we have considered in this work, $k_{x} \ll k_{y}$, motions are essentially incompressible. A clear example of this situation is the kink mode in the compressible slab (see Arregui et al. 2007) or the kink mode in cylindrical geometry in the thin tube limit (see Goossens et al. 2009). Hence, incompressibility is a reasonable approximation that has the additional advantage that the problem can be studied fully analytically, this has allowed us to derive the algebraic dispersion relation given by Eq. (22). Notice also that the choice of a step function for the density is convenient to avoid a situation where the Alfvén speed smoothly changes with height, since this would imply the existence of resonances for the case with $k_{y} \neq 0$.

From the analysis of the dispersion relation and the eigenfunctions we have shown that, contrary to the purely magnetic case, the global transverse kink mode does not necessarily exist for the whole range of parameters in the stratified model, even in the stable regime. This might have some important consequences, since other types of motions associated to the individual interfaces, or of mixed nature between surface and global kink waves, are feasible according to our model. Thus, from the observational point of view it might be interesting to try to identify such kind of motions since their detection can give more clues about the real physical conditions in threads, specially about the interplay between magnetic and gravitational forces.

Using the slab model we have demonstrated that the system allows a mode that is always stable, independently of the value of the Alfvén speed in the thread, while there is another mode that is Rayleigh-Taylor unstable but becoming stable when the Alfvén speed is increased. Hence, gravity might have a strong effect on the modes of oscillation of horizontal threads, depending on the strength of the magnetic field. In the case of threads in quiescent filaments like the one studied by Lin et al. (2007), where the Alfvén speed presumably low, too short lifetimes are found using the slab model, i.e., the instability is too efficient. On the contrary, in active region prominences (like in Okamoto et al. 2007), the stabilising effect of the magnetic tension might be enough to suppress the Rayleigh-Taylor instability for a wide range of wavelengths. We have also shown that the instability can be used to establish the minimum Alfvén speed in the thread, an approach that is different from other seismological analysis of thread oscillations (see for example Terradas et al. 2008; Lin et al. 2009; Soler et al. 2010). Nevertheless, more elaborated models need to be explored in detail before a reliable comparison of periods and thread lifetimes with observations can be performed.

Finally, we have to remark that the classification of the modes and their spatial structure strongly depends on the slab configuration. As we have already mentioned, the sausage mode found in our system when the magnetic field is dominant does not exist as a trapped mode in the cylindrical tube. This mode is precisely becoming unstable when gravity dominates over the magnetic field. This means that the geometry can have an important effect on the spectrum of the modes, and a careful analysis using more realistic geometries (see Blokland \& Keppens 2011b, for flattened cylindrical models) is required to understand well the effect of gravity in horizontal threads.

Acknowledgements. J.T. acknowledges support from the Spanish Ministerio de Educación y Ciencia through a Ramón y Cajal grant. All the authors acknowledge the funding provided under the project AYA2011-22846 by the Spanish MICINN/MINECO and FEDER Funds. The financial support from CAIB through the "Grups Competitius" scheme and FEDER Funds is also acknowledged. The authors also thank I. Arregui, A. J. Díaz and R. Soler for their comments and suggestions that helped to improve the paper.

\section{References}

Arregui, I., Terradas, J., Oliver, R., \& Ballester, J. L. 2007, Sol. Phys., 246, 213 Arregui, I., Terradas, J., Oliver, R., \& Ballester, J. L. 2008, ApJ, 682, L141 Arregui, I., Soler, R., Ballester, J. L., \& Wright, A. N. 2011, A\&A, 533, A60 Arregui, I., Oliver, R., \& Ballester, J. L. 2012, Liv. Rev. Sol. Phys., 9, 2 
Banerjee, D., Erdélyi, R., Oliver, R., \& O'Shea, E. 2007, Sol. Phys., 246, 3 Berger, T. E., Shine, R. A., Slater, G. L., et al. 2008, ApJ, 676, L89 Berger, T. E., Slater, G., Hurlburt, N., et al. 2010, ApJ, 716, 1288

Blokland, J. W. S., \& Keppens, R. 2011a, A\&A, 532, A93

Blokland, J. W. S., \& Keppens, R. 2011b, A\&A, 532, A94

Cally, P. S. 1986, Sol. Phys., 103, 277

Cally, P. S. 2003, Sol. Phys., 217, 95

Carbonell, M., Oliver, R., \& Ballester, J. L. 2004, A\&A, 415, 739

Chae, J., Ahn, K., Lim, E.-K., Choe, G. S., \& Sakurai, T. 2008, ApJ, 689, L73

Chandrasekhar, S. 1961, Hydrodynamic and hydromagnetic stability

Díaz, A. J., Oliver, R., Erdélyi, R., \& Ballester, J. L. 2001, A\&A, 379, 1083

Díaz, A. J., Oliver, R., \& Ballester, J. L. 2002, ApJ, 580, 550

Díaz, A. J., Oliver, R., \& Ballester, J. L. 2005, A\&A, 440, 1167

Dymova, M. V., \& Ruderman, M. S. 2005, Sol. Phys., 229, 79

Edwin, P. M., \& Roberts, B. 1982, Sol. Phys., 76, 239

Edwin, P. M., \& Roberts, B. 1983, Sol. Phys., 88, 179

Goedbloed, J. P. H., \& Poedts, S. 2004, Principles of Magnetohydrodynamics

Goossens, M., Terradas, J., Andries, J., Arregui, I., \& Ballester, J. L. 2009, A\&A, 503,213

Hermans, D., \& Goossens, M. 1987, A\&A, 172, 85

Hermans, D., \& Goossens, M. 1989, A\&A, 225, 569

Hillier, A., Isobe, H., Shibata, K., \& Berger, T. 2011, ApJ, 736, L1

Hillier, A., Berger, T., Isobe, H., \& Shibata, K. 2012, ApJ, 746, 120

Joarder, P. S., Nakariakov, V. M., \& Roberts, B. 1997, Sol. Phys., 173, 81

Lerche, I., \& Low, B. C. 1980, Sol. Phys., 67, 229
Lin, Y. 2011, Space Sci. Rev., 158, 237

Lin, Y., Engvold, O., Rouppe van der Voort, L., Wiik, J. E., \& Berger, T. E. 2005, Sol. Phys., 226, 239

Lin, Y., Engvold, O., Rouppe van der Voort, L. H. M., \& van Noort, M. 2007, Sol. Phys., 246, 65

Lin, Y., Soler, R., Engvold, O., et al. 2009, ApJ, 704, 870

Low, B. C., \& Zhang, M. 2004, ApJ, 609, 1098

McEwan, M. P., \& Díaz, A. J. 2007, Sol. Phys., 246, 243

Okamoto, T. J., Tsuneta, S., Berger, T. E., et al. 2007, Science, 318, 1577

Oliver, R., \& Ballester, J. L. 2002, Sol. Phys., 206, 45

Petrie, G. J. D., Blokland, J. W. S., \& Keppens, R. 2007, ApJ, 665, 830

Rayleigh, L. 1883, Proc. London Mathematical Society, 14, 170

Ryutova, M., Berger, T., Frank, Z., Tarbell, T., \& Title, A. 2010, Sol. Phys., 267,

Soler, R. 2010, Ph.D. Thesis, Departament de Fisica, Universitat de les Illes Balears

Soler, R., Arregui, I., Oliver, R., \& Ballester, J. L. 2010, ApJ, 722, 1778

Soler, R., Ballester, J. L., \& Goossens, M. 2011, ApJ, 731, 39

Soler, R., Ballester, J. L., \& Parenti, S. 2012, A\&A, 540, A7

Spruit, H. C. 1982, Sol. Phys., 75, 3

Taylor, G. 1950, Roy. Soc. London Proc. Ser. A, 201, 192

Terradas, J., Arregui, I., Oliver, R., \& Ballester, J. L. 2008, ApJ, 678, L153

Thompson, W. T., \& Schmieder, B. 1991, A\&A, 243, 501

Yi, Z., \& Engvold, O. 1991, Sol. Phys., 134, 275

Yi, Z., Engvold, O., \& Keil, S. L. 1991, Sol. Phys., 132, 63 